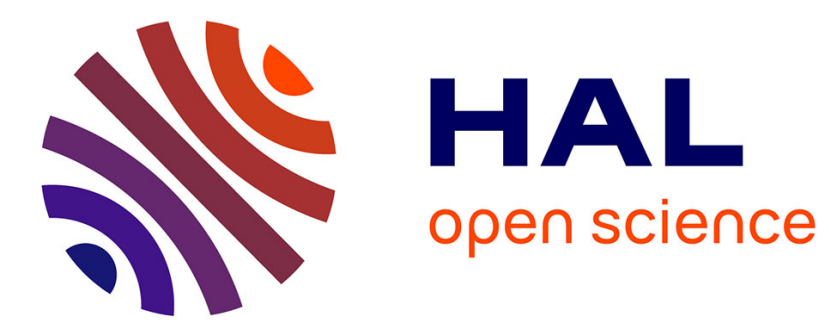

\title{
Measurements and correlations for gas liquid surface tension at high pressure and high temperature
}

Clément Leonard, Jean-Henry Ferrasse, Olivier Boutin, Sébastien Lefevre, Alain Viand

\section{- To cite this version:}

Clément Leonard, Jean-Henry Ferrasse, Olivier Boutin, Sébastien Lefevre, Alain Viand. Measurements and correlations for gas liquid surface tension at high pressure and high temperature. AIChE Journal, 2018, 64 (11), pp.4110-4117. 10.1002/aic.16216 . hal-02114480

\section{HAL Id: hal-02114480 \\ https://hal-amu.archives-ouvertes.fr/hal-02114480}

Submitted on 29 Apr 2019

HAL is a multi-disciplinary open access archive for the deposit and dissemination of scientific research documents, whether they are published or not. The documents may come from teaching and research institutions in France or abroad, or from public or private research centers.
L'archive ouverte pluridisciplinaire HAL, est destinée au dépôt et à la diffusion de documents scientifiques de niveau recherche, publiés ou non, émanant des établissements d'enseignement et de recherche français ou étrangers, des laboratoires publics ou privés. 
Measurements and Correlations for Gas Liquid Surface Tension at High Pressure and High Temperature

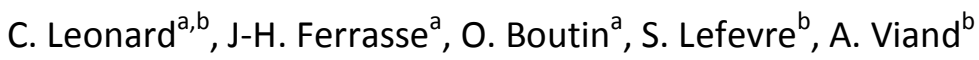

a Aix Marseille Univ, CNRS, Centrale Marseille, M2P2, Marseille, France

b S.A.R.L. A3i; 255 rue Gustave Eiffel, ZAC des Eoliennes; 26290 Donzere, France

\begin{abstract}
Surface tension of water/nitrogen and water-phenol/nitrogen systems was successfully measured by the hanging drop method in a wide domain of temperature (from 100 to $300^{\circ} \mathrm{C}$ ) and pressure (from 4 to $30 \mathrm{MPa}$ ), conditions little explored literature. Results show that surface tension of water-phenol mixtures decreases as phenol mass fraction increases. This decrease is observed under saturated and unsaturated conditions and is more pronounced at low temperatures and does not seem to depend on pressure. The effect of saturation on surface tension in the water/nitrogen system has been correlated with water vapor pressure by using experimental points with a great accuracy. For the waterphenol/nitrogen system, experimental data obtained with different mass fraction of phenol were correlated using Macleod-Sugden equation for mixtures.
\end{abstract}

\title{
Topical heading
}

Reaction Engineering, Kinetics and Catalysis

Transport Phenomena and Fluid Mechanics 
Key words: surface tension, bubble column, high pressure, high temperature

\section{Introduction}

Multiphase gas-liquid-solid contactors are often used in chemical engineering processes. For instance, the gas phase can be dispersed into the liquid or suspension in the form of bubbles using a gas sparger generally placed at the bottom of a column. The so called "bubble column" can work in semi-batch mode (batch for liquid) or in continuous mode. This kind of reactor are generally used in chemical, biochemical, petroleum and metallurgical industries (oxidation, polymerisation, hydrogenation, gas conversion to produce fuels, fermentation, biological wastewater treatment ....). For instance, the Fischer-Tropsch synthesis is carried on bubble columns and is widely studied in the literature. ${ }^{1,2,3,4}$ Several parameters influence the operating of bubble columns: gas-liquid system, pressure, temperature, gas and liquid superficial velocities, operating mode, design parameters and physical properties. $5,6,7,8,9,10,11,12,13,14$ The hydrodynamics of gas liquid contactor is not fully known and difficult to predict. Indeed, most of the studies only focus on two to four parameters and are depending on the system. Moreover, many data correlations exist for atmospheric and low pressure and temperature, but very few at high pressure and high temperature. 1,15,16,8,17,18,19,20,21,22,23,24,25 To better understand the behaviour of gas liquid systems at high pressure and temperature, it is first necessary to know the evolution of physical properties in these conditions. The effect of pressure, temperature and saturation on surface tension is investigated in this article.

Surface tension values for water at high temperature up to the critical point, in balance with its vapor, are well known. ${ }^{26,27}$ However, few data are available in respect of pressure effect on surface tension of water/nitrogen and water/air mixtures. Yi-Ling et al. ${ }^{28}$ have measured 
surface tension of water in the presence of various gases and liquids $\left(\mathrm{N}_{2}, \mathrm{O}_{2}, \mathrm{n}\right.$-hexane, $\mathrm{n}$ heptane, $\mathrm{CH}_{4}$ ) at pressures up to $100 \mathrm{MPa}$ and temperatures up to $200^{\circ} \mathrm{C}$ by the hanging drop method. This is the only available publication providing data for water behaviour at high pressures. The estimation of surface tension under high pressure and high temperature can be difficult because of the aqueous solutions behaving in a non-ideal way under pressure. Experimental values of these parameters are not available in the literature and must therefore be determined either by correlations or by direct measurements. In this study, surface tensions of water and water-phenol solutions were measured with nitrogen.

Available scientific literature proposes several experimental methods for the measurement of surface tension: Wilhelmy's blade, Noüy's Lecomte ring, maximum drop pressure, capillary ascension or depression, rotating drop, hanging drop and drop weight. ${ }^{29,30,31}$ Noüy's Ring and capillary ascension are known to be complex at high temperatures. The method of rotating drop cannot be run under pressure. The method of Wilhelmy's blade requires a very specific apparatus. The method of maximum drop pressure uses tables whose validity is not verified at high pressure. At high pressure and high temperature, the method commonly used is the hanging drop method. ${ }^{25,28,32,33,34,35,36,37,38,39,40,41,42,49}$. The sessile drop method is sometimes used. ${ }^{43}$ Considering pressure constraints, it seems easier to stabilize a drop at the end of a capillary, in an autoclave (hanging drop), than to settle a drop on a stainless-steel surface, in the case of sessile drop. Another advantage of the hanging drop method is its accuracy using Misak tables. It can also be used under saturated and unsaturated conditions, provided that the saturation is slower than the measurement time. Because of above comments, the hanging drop method is chosen for this study. 


\section{Materials and Methods}

\section{Surface tension calculation}

In 1806, Laplace established the relation between the pressure gradient on either side of any surface and the curvature of the interface. For drops or bubbles symmetrical regarding the vertical axis, the pressure difference $\Delta \rho g$ can be expressed by Laplace-Young equation (equation 1$). .^{32,33}$

$$
\Delta \rho g h=\sigma_{L}\left(\frac{1}{\mathcal{R}_{1}}+\frac{1}{\mathcal{R}_{2}}\right)
$$

$\mathcal{R}_{1}$ et $\mathcal{R}_{2}$ are the radii of curvature at a point at intersection of two orthogonal area of the surface. This equation indicates that knowledge of the radius of curvature and therefore of the shape of the drop or bubble is sufficient to determine the surface tension. The radii of curvature are difficult to determine: by expressing them according to the $\mathrm{x}$ and $\mathrm{z}$ coordinates of the profile, a differential equation presenting no simple analytical solution is obtained. However, a numerical solution of the Laplace-Young equation has been obtained by Bashforth and Adams. ${ }^{44}$ In the case of a hanging drop, presented in Figure 1 , the Bashforth and Adams equation can be obtained by setting $x / \sin \varphi=\mathcal{R}_{2}$ and introducing this term into the Laplace-Young equation. It is given by Eq. 2 .

$$
\begin{aligned}
& \frac{1}{\frac{\mathcal{R}_{1}}{\mathcal{R}_{\text {apex }}}}+\frac{\sin \varphi}{\frac{x}{\mathcal{R}_{\text {apex }}}}=\beta_{0} \frac{z}{\mathcal{R}_{\text {apex }}}+2 \\
& \text { with } \beta_{0}=\frac{\Delta \rho g \mathcal{R}_{\text {apex }}{ }^{2}}{\sigma_{L}}
\end{aligned}
$$

The formulation of Bashforth and Adams is equivalent to the Laplace's law. The resolution of Eq. 2 is done using the tables established by Bashforth and Adams, ${ }^{44}$ which provide the values of $x / \mathcal{R}_{\text {apex }}$ by knowing $z / \mathcal{R}_{\text {apex }}$ and $\beta_{0}$ (in the range 0.125 to 100 ). A fast resolution 
uses form factors $F_{1}$ and $F_{2}$ (Eq. 4) involving the characteristic diameters of the drop (Figure 1). ${ }^{46}$

$$
F_{1}=\frac{d_{s}}{d_{e}} \quad F_{2}=\beta_{0}\left(\frac{d_{e}}{\mathcal{R}_{\text {apex }}}\right)^{2}
$$

Surface tension can be expressed according to Eq. 5:

$$
\sigma_{L}=\frac{\Delta \rho g d_{e}^{2}}{F_{2}}
$$

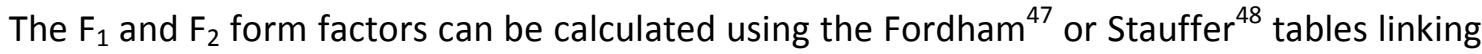
$1 / F_{2}$ to $F_{1}$. However, it is simpler to use the Misak equations ${ }^{50}$ linking $1 / F_{2}$ to $F_{1}$ for different $F_{1}$ intervals (Appendix 1).

Water and nitrogen densities are provided by the NIST database. ${ }^{26}$ Under unsaturated conditions, water and nitrogen densities will be settled at operating process pressure and temperature. Under saturated conditions, the water is in equilibrium with its vapor phase (also given by NIST as a function of temperature). This vapor pressure will be settled equal to the partial pressure of water in the gas phase. The density of the gas phase is calculated by Eq. 6 .

$$
\rho_{G, s a t}(T, P)=\left(1-y_{v, H 2 O}\right) \rho_{N 2}(T, P)+\rho_{v a p}\left(T, P_{v a p}\right)
$$

Water density under saturated conditions will be chosen equal to the water density in equilibrium with its vapor at the considered temperature. In the case of aqueous solutions of phenol, the amount of phenol present in the gas phase will be neglected and the properties of the water will be taken for the liquid phase. This approximation is valid at low phenol content in water. 
Furthermore, the method of the hanging drop is not very precise for low (less than $0.3^{45}$ ) or large $\left(\approx 10^{51}\right)$ Bond numbers for which the drop profile is close to a sphere. ${ }^{37,45,51,52}$ It is therefore necessary to work at intermediate conditions of Bond number. Saad et al. ${ }^{51}$ have shown that Bond number calculation according to the analysis of Padday and $\mathrm{Pitt}^{53}$ allows to correctly represent the results in terms of critical Bond number. The modified Bond number can be estimated by Eq. 7 .

$$
\begin{aligned}
& B o=\left(\frac{r_{c}}{\alpha}\right)^{2}=\frac{\Delta \rho g r_{c}{ }^{2}}{\sigma_{L}} \\
& \text { with } \alpha=\sqrt{\frac{\sigma_{L}}{\Delta \rho g}}
\end{aligned}
$$

The term $\alpha$, defined by Eq. 8, represents the capillary length. By developing a criterion based on drops volume, the authors showed that the Bond number range required to obtain a satisfactory accuracy varied between $10^{-2}$ and $3 .{ }^{51}$ In the systems studied, the difference in densities can vary between 400 and $1000 \mathrm{~kg} \cdot \mathrm{m}^{-3}$ and the surface tension is likely to vary between 10 and $75 \mathrm{mN} . \mathrm{m}^{-1}$. A capillary with an outer diameter of $1 / 16^{\prime \prime}(1.5 \mathrm{~mm})$ and an internal diameter of $1 / 64 "(0.5 \mathrm{~mm})$ was chosen to obtain modified Bond numbers between 0.096 and 0.2 . This capillary is identical to the one used by Chiquet et al. ${ }^{37}$

\section{Experimental measurements}

The general layout of the installation is shown in Figure 2. The reactor is supplied with gas $\left(N_{2}\right)$ via a 20MPa pressurized gas cylinder supplied by AIR LIQUIDE (purity > 99\%). The positive displacement pump supplied by TOP INDUSTRIE has a volume of $50 \mathrm{~cm}^{3}$. The liquid circuit is connected to the capillary and the gas circuit is directly connected to the reactor. The reactor is a $316 \mathrm{~L}$ stainless steel autoclave with an internal volume of $150 \mathrm{~cm}^{3}$ made by TOP INDUSTRIE $\left(38 \mathrm{MPa}, 400^{\circ} \mathrm{C}\right)$. It is equipped with a ceramic band heater and a double 
jacket in which circulates a cooling fluid. A thermocouple is inserted into the reactor core via an inlet at the bottom of the reactor. The capillary system consists of a $1 / 4^{\prime \prime}(6.35 \mathrm{~mm})$ external diameter stainless steel tube into which the $1 / 8 \mathrm{in}$. $(3.175 \mathrm{~mm})$ outer diameter capillary is immersed. Visualization of the drops is done thanks to two sapphire windows. Two Inconel rings between the windows and the reactor (Figure 3) seal the autoclave. Drops pictures were made in using a camera with a $19 \mathrm{MPixel}$ resolution and equipped with a $60 \mathrm{~mm}$ macro lens with fixed focal length $(f / 2.8)$. Considering an uncertainty of two pixels for the measurement of a diameter, these are determined to $\pm 22 \mu \mathrm{m}$.

Experiments are performed according to the protocol described below.

- The reactor and the pump circuit are purged with a nitrogen stream to remove any traces of water and/or phenol in the system (at ambient pressure and at $200^{\circ} \mathrm{C}$ ).

- A low nitrogen pressure (see calculation in Appendix 2) is loaded into the reactor. While increasing temperature during heating, the pressure in the reactor will increase until reaching the set point.

- The circuit of the pump is then washed with the liquid. It is then injected into the capillary after pressurising the liquid circuit at the working pressure.

- The liquid is pumped at a rate of $0.00025 \mathrm{~cm}^{3} \cdot \mathrm{s}^{-1}$ to slowly form a droplet at the end of the capillary. The pump is then shut down and the droplet is photographed. Five to twenty successive drops are then photographed. As the drops fall directly on the thermocouple, it can be considered that the drop is at the reactor setpoint temperature if it does not cause a change in the value read by the thermocouple. The evolution of the interfacial tension with the progressive saturation of the medium is evaluated by analysing the surface tension of the successive drops. It is possible to verify the hypothesis of unsaturation by comparing the 
surface tension values of the first drops obtained with those obtained under intermediate conditions and complete saturation. Direct visualization shows that the liquid layer formed at the bottom of the reactor gradually evaporates. During the saturation step the pressure increases in the reactor. The pressure is therefore regularly adjusted by the gas purge valve. The saturation point is reached when the first drop of liquid is formed at the sapphire wall. Once this point is reached, several drops are also measured.

Measurements are carried out in a pressure range of 4 to $30 \mathrm{MPa}$, and temperatures between 100 and $300^{\circ} \mathrm{C}$. The mass fraction of phenol in the aqueous phase was tested between 0.02 and 0.08 . This corresponds to phenol mass concentrations between 20 and $80 \mathrm{~g} . \mathrm{L}^{-1}$, under ambient conditions of pressure and temperature. The uncertainty on temperature is about $0.1^{\circ} \mathrm{C}$. The uncertainty of the surface tension can be evaluated by calculating the experimental standard deviation from several drops (between 3 and 16 depending of the manipulation). Standard deviations obtained by these calculations vary from \pm 0.48 to $\pm 2.21 \mathrm{mN} \cdot \mathrm{m}^{-1}$, leading to percentages of uncertainties between 0.9 and $6.3 \%$ with an average of $3.2 \%$. Uncertainties do not depend on temperature, pressure, and saturation conditions.

\section{Results and discussion}

At first, to validate the experimental setup, results with water/nitrogen system are presented.

\section{Water/nitrogen system}

Two reproducibility tests in unsaturated conditions are presented in Table 1 . Results indicate that the uncertainties on the average of the two tests are lower than those 
calculated from several drops during the same manipulation. The measurement is reproducible.

The results obtained in the Water/Nitrogen system for all pressure and temperature conditions are shown in Figure 4 with saturation NIST data ${ }^{26,27}$ and results of Yi-Ling et al. ${ }^{28}$

Figure 4 shows that measurements under saturated conditions agree with NIST database values. ${ }^{26,27}$ The deviation with literature values vary between 0.4 and $7.2 \%$ with only 2 data having more than $4 \%$ of difference $\left(10 \mathrm{MPa}-160^{\circ} \mathrm{C}\right.$ and $\left.30 \mathrm{MPa}-300^{\circ} \mathrm{C}\right)$. The experimental setup is then validated under saturated conditions. The NIST data does not take pressure into account. In the tests performed in this study, measurements under saturated conditions show that pressure has a little effect (data at $100^{\circ} \mathrm{C}$ and 0.1013 and $4 \mathrm{MPa}, 2 \%$ uncertainty on the average). This is the same for the measurements performed under unsaturated conditions. For instance, the data at 10 and $15 \mathrm{MPa}$ for a temperature of $160^{\circ} \mathrm{C}$ have only $2.5 \%$ difference. The data at $10,15,20$ and $25 \mathrm{MPa}$ for a temperature of $220^{\circ} \mathrm{C}$ have a standard deviation of 0.22 or an uncertainty of $0.6 \%$. It is then surprising to note that the results of $\mathrm{Yi}$-Ling et al. ${ }^{28}$ show a significant pressure effect, especially at low temperatures below $200^{\circ} \mathrm{C}$. They observe a decrease in surface tension as pressure increases. This could indeed be observed on our measurements comparing the results obtained at 160 and at $100^{\circ} \mathrm{C}$ for which the same trend is postponed. Assuming that the measurements of Yi-Ling et al. ${ }^{28}$ are performed under saturated and high-pressure conditions, their experiments are then in agreement with our measurements and with this trend. Figure A3 in Appendix 3 illustrates these results. This pressure effect on surface tension seems to present a plateau at the highest pressures. It is not observed significantly at temperatures of $200^{\circ} \mathrm{C}$ by Yi-Ling et al..$^{28}$ 
The effect of medium saturation is not negligible. Figure 5 represents the evolution of surface tension as a function of the number of drops analysed, for an experiment at $30 \mathrm{MPa}$ and $300^{\circ} \mathrm{C}$. It shows that surface tension begins to decrease after about 5 to 6 drops analysed. The medium is considered unsaturated before this point. At $100^{\circ} \mathrm{C}$, the difference between measurements under dry and saturated conditions is of the order of magnitude of the measurement uncertainty. However, this difference increases at $150^{\circ} \mathrm{C}$ and becomes significant at $220^{\circ} \mathrm{C}$. It is possible to represent the difference between measurements in dry conditions and in unsaturated conditions as a function of temperature, using NIST data as a reference (Appendix 4 Figure A2). An uncertainty on vapor pressure of $0.025 \%$ is provided by NIST. The difference between values under dry conditions and saturated conditions increases with temperature according to a power law. Uncertainties about the measured difference are also represented. It is also noted that deviation from saturation is very similar to the increase of saturation vapor pressure of water. The effect of saturation can therefore be directly correlated from this parameter, assuming a linear law between saturation vapor pressure and saturation deviation and neglecting the effect of total pressure (Eq. 9).

$$
\frac{\sigma_{L, d r y}}{\sigma_{L, s a t}}=1+C P_{\text {sat }, H 2 O} \text { with } \mathrm{C}=6,090.10^{-8} \pm 4,315.10^{-9} \mathrm{~Pa}^{-1}
$$

The prediction is carried out with an average uncertainty of $2.4 \%$ with our experimental points, the highest differences being obtained at temperatures of 100 and $160^{\circ} \mathrm{C}$ for which the possible effect of the pressure is not considered. Equation 9 can be used to calculate surface tension of systems operating under unsaturated conditions and for which no surface tension data are available. The experimental setup was validated by measurements on the water/nitrogen system. The measurements on waterphenol/nitrogen system are presented below. 


\section{Water-phenol/nitrogen system}

The results obtained in the water-phenol/nitrogen system are shown in Figure 6 for the 4 phenol mass fractions (0/0.022/0.041/0.061/0.081). Figure 6 indicates that surface tension of water-phenol mixtures decreases as phenol mass fraction increases. This decrease is observed under saturated and unsaturated conditions and is more pronounced at low temperatures. It does not seem to depend on pressure. Thus, the maximum deviations between values for pure water and those for a phenol mass fraction of 0.081 under unsaturated conditions are higher at $100^{\circ} \mathrm{C}\left(38 \%\right.$ decrease in surface tension) than at $300^{\circ} \mathrm{C}$ (12\% decrease). The differences are substantially identical for trials performed at $220^{\circ} \mathrm{C}$ at 10 and $20 \mathrm{MPa}$ (20 and $22 \%$ decrease respectively). In saturated conditions the same tendencies are observed but the relative decreases are smaller (for example: $4 \mathrm{MPa}-100^{\circ} \mathrm{C}$ : $\left.36 \%, 10 \mathrm{MPa}-220^{\circ} \mathrm{C}: 14 \%, 30 \mathrm{MPa}-300^{\circ} \mathrm{C}: 11 \%\right)$. The effect of pressure was only tested at $220^{\circ} \mathrm{C}$. The influence of pressure does not seem to depend on phenol mass fraction. It can be noted that, for mass fractions between 0.022 and 0.081 , the difference between the values obtained at 10 and $20 \mathrm{MPa}$ decreases as phenol mass fraction increases under saturated and unsaturated conditions. However, the maximum difference is obtained for a phenol mass fraction of $0.022(6 \%)$, under saturated and unsaturated conditions. the other differences being below $3 \%$. This high difference at 0.022 is not concluding and can be due to partial saturation of the medium at the beginning of one of the test.

Equation 9 was tested for the water/phenol system using the same constant $C$ and water saturation pressure. The adequacy between the results measured in dry conditions and those calculated by equation 9 from the measurements made under saturated conditions is correct (Appendix 5 Figure A3). Therefore, this simple equation makes possible to correctly 
represent the results of water-phenol/nitrogen system. It is useful to determine the surface tension of a liquid system under unsaturated conditions from the knowledge of a system under saturated conditions. A correlation for measurements under saturated conditions must therefore be proposed. The literature proposes four correlations for estimating surface tension of mixtures: Macleod and Sugden, Szyszkowski, Tamura and Suarez. ${ }^{54}$ For aqueous binary systems including water and an organic molecule, the Tamura and Suarez methods are generally recommended as they can predict non-linear trends in the influence of the molar fraction of organic compound in water (see Figure 6). However, not all data is known to use these methods. Szyszkowski's relation is valid under very dilute conditions ${ }^{54}$, which is not the case in this study. It is therefore possible to test only the validity of the Macleod-Sugden correlation for mixtures. This is in the form of Eq. 10 with the mixing rules proposed by Hugill and Van Welsenes. ${ }^{55}$

$$
\sigma_{L, m}=\left(\left[P_{L, m}\right] \rho_{L, m}-\left[P_{G, m}\right] \rho_{G, m}\right)^{C}
$$

The term $\left[P_{\mathrm{i}, \mathrm{m}}\right]$ represents the Parachor of the liquid or the gaseous mixture. These numbers can be determined by Eqs. 11 to 13.

$$
\begin{aligned}
& {\left[P_{L, m}\right]=\sum_{i} \sum_{j} x_{i} x_{j}\left[P_{i, j}\right]} \\
& {\left[P_{G, m}\right]=\sum_{i} \sum_{j} y_{i} y_{j}\left[P_{i, j}\right]} \\
& {\left[P_{i, j}\right]=\delta_{i j} \frac{\left[P_{i}\right]+\left[P_{j}\right]}{2}}
\end{aligned}
$$

The term $\left[P_{\mathrm{i}}\right]$ represents the Parachor of compound i. assumed constant with temperature and pressure. The term $\delta_{i j}$ is a binary interaction parameter to be determined by fitting with experimental data. Note that the definition and calculation of Parachor assume that 
the surface tension is independent of the surrounding gas. The liquid is supposed to be in equilibrium with its vapor. To verify the Macleod-Sugden correlation for saturated experiments, the Parachor of water and phenol were estimated. The water Parachor was determined from surface tension and molar volume data from NIST. The calculation is presented in Appendix 6 (52.5). The phenol Parachor can be calculated by a group contribution method but a publication provides its value, calculated from measurements of surface tension up to $150^{\circ} \mathrm{C}(224.8) .{ }^{56}$ Their results show that the phenol Parachor varies with temperature and increases very slightly with increasing temperature. Under these conditions, the numerical value is chosen at an average value in the temperature range studied. The dew point composition of the water-phenol system should also be estimated. For this purpose, ProSim ${ }^{\circledR}$ software (France) is used for calculation. Thermodynamic model used is the Predictive Soave-Redlich-Kwong (PSRK) state equation with PSRK mixing rules and UNIFAC-PSRK model for estimating activity coefficients. The initial mass fraction of the mixture $(0.022 / 0.041 / 0.061 / 0.081)$ is set as well as temperature. The software then calculates vapor pressure of the mixture and the molar compositions of water and phenol in the gas and liquid phases (Appendix 7). The model predicts an identical gas phase molar composition regardless of the temperature of the mixture. The vapor pressure of waterphenol mixture is substantially identical to that of pure water. A slight increase $(2 \%$ maximum difference/water, at $300^{\circ} \mathrm{C}$, less than $1 \%$ at other temperatures) is observed when phenol mass fraction increases. It is however more convenient to use a correlation using the properties of water than the properties of the mixture, especially since measurement uncertainty does not justify such precision on vapor pressure. The molar fractions in the liquid and gas phase being low, the pure water densities are taken for the calculation. Predictions of the Macleod-Sugden equation for mixtures are shown in Figure 7. 
Predictions of the Macleod-Sugden correlation are mostly correct. The data at 4 and $20 \mathrm{MPa}$ are well predicted. Values at 25 and 30MPa are overestimated with differences between 7.5 and $13 \%$. The point obtained at $10 \mathrm{MPa}$ and an $x_{\mathrm{w}}$, Ph of 0.022 is the worst predicted. The average error obtained is $6 \%$ with an average difference of $\pm 1.6 \mathrm{mN} . \mathrm{m}^{-1}$. This correlation, among the simplest to predict the surface tension, is hence validated. The interaction parameters and the exponent $\mathrm{C}$ are given in Table 2.

These coefficients are valid in with a phenol mass fraction in the range 0.02 to 0.08 . Indeed, it has been tested to extrapolate the correlation up to $x_{\mathrm{w}}$, ph $=0$ but the prediction of equation 12 becomes mediocre. The value of 3.36 of the exponent agrees with the results of

the literature for other systems. ${ }^{54}$ Finally, it should be noted that calculation for molar fractions in the liquid phase of less than 0.01 by Szyszkowski's correlation are less satisfactory than those of the Macleod-Sugden correlation.

\section{Conclusions}

Two sets of experimental data have been obtained during this work on the surface tension of water/nitrogen and water-phenol/nitrogen systems by using the hanging drop method. This is an important point as very few data are available in the studied domain of pressure (15 to $30 \mathrm{MPa})$ and temperature $\left(100\right.$ to $\left.300^{\circ} \mathrm{C}\right)$. Experimental points obtained in the water / nitrogen system, in saturated and unsaturated conditions, are directly correlated to the saturation vapor pressure by a linear law, neglecting the effect of the total pressure, with an average uncertainty of $2.4 \%$. The established correlation can be used to calculate the surface tension of systems operating under unsaturated conditions from saturated data (obtain in databases such as NIST). Tests on the water-phenol / nitrogen system show that 
the surface tension of the water-phenol mixture decreases as the phenol mass fraction increases. The correlation established for the effect of saturation on the water / nitrogen system is also valid for the water-phenol/nitrogen system. To correlate surface tension values under saturated conditions in the studied domain, the experimental values were subjected to the Macleod-Sugden correlation, with mixing rules integrating the calculation of Parachor. The proposed coefficients (interaction parameters of the Parachor, constant of the linear law between the surface tension and the saturation vapor pressure) makes it possible to estimate with a good precision the surface tension of the saturated mixtures Water-Phenol/Nitrogen in the fraction range phenol mass of 0.02 to 0.08 . 


\section{Literature Cited}

1. Behkish A, Men Z, Inga JR, and Morsi, BI. Mass transfer characteristics in a large-scale slurry bubble column reactor with organic liquid mixtures. Chemical Engineering Science. 2002;57:3307-3324.

2. Deckwer WD., Louisi L, Zaidi A, Ralek. Hydrodynamic Properties of the Fischer-Tropsch Slurry Process. Industrial \& Engineering Chemistry Process Design and Development. 1980;19:699-708.

3. Gandhi B, Prakash A, Bergougnou MA. Hydrodynamic behavior of slurry bubble column at high solids concentrations. Powder Technology. 1999;103:80-94.

4. Krishna R, Sie ST. Design and scale-up of the Fischer-Tropsch bubble column slurry reactor. Fuel Processing Technology. 2000;64:73-105.

5. Chilekar VP, Van der Schaaf J, Kuster BFM, Tinge JT, Schouten JC. Influence of elevated pressure and particle lyophobicity on hydrodynamics and gas-liquid mass transfer in slurry bubble columns. AIChE Journal. 2010;56:584-596.

6. Gourich B, Vial C, Essadki AH, Allam F, Belhaj SM, Ziyad M. Identification of flow regimes and transition points in a bubble column through analysis of differential pressure signalInfluence of the coalescence behavior of the liquid phase. Chemical Engineering and Processing: Process Intensification. 2006;4:214-223.

7. Grover GS, Rode CV, Chaudhari RV. Effect of temperature on flow regimes and gas holdup in a bubble column. The Canadian Journal of Chemical Engineering. 1986;64:501-504. 
8. Hashemi S, Macchi A, Servio P. Gas-liquid mass transfer in a slurry bubble column operated at gas hydrate forming conditions. Chemical Engineering Science. 2009;64:37093716.

9. Kemoun A, Cheng Ong B, Gupta P, Al-Dahhan MH, Dudukovic MP. Gas holdup in bubble columns at elevated pressure via computed tomography. International Journal of Multiphase Flow. 2001;27:929-946.

10. Letzel MH, Schouten JC, Van den Bleek CM, Krishna R. Effect of gas density on largebubble holdup in bubble column reactors. AIChE Journal. 1998;44:2333-2336.

11. Şal S, Gül ÖF, Özdemir M. The effect of sparger geometry on gas holdup and regime transition points in a bubble column equipped with perforated plate spargers. Chemical Engineering and Processing: Process Intensification. 2013;70:259-266.

12. Thorat BN, Joshi JB. Regime transition in bubble columns: experimental and predictions. Experimental Thermal and Fluid Science. 2004;28:423-430

13. Vial C, Poncin S, Wild G, Midoux N. A simple method for regime identification and flow characterisation in bubble columns and airlift reactors. Chemical Engineering and Processing: Process Intensification. 2001;40:135-151.

14. Zahradník J, Fialová $M$, Růžička $M$, Drahoš J, Kaštánek $F$, Thomas NH. Duality of the gasliquid flow regimes in bubble column reactors. Chemical Engineering Science. 1997;52:38113826.

15. Clark KN. The effect of high pressure and temperature on phase distributions in a bubble column. Chemical Engineering Science. 1990;45:2301-2307. 
16. Fan LS, Yang GQ, Lee DJ, Tsuchiya K, Luo X. Some aspects of high-pressure phenomena of bubbles in liquids and liquid-solid suspensions. Chemical Engineering Science. 1999;54:4681-4709.

17. Idogawa K, Ikeda K, Fukuda T, Morooka S. Effects of Gas and Liquid Properties on the Behavior of Bubbles in a Bubble Column under High Pressure. Kagaku Kogaku Ronbunshu. 1985;11:432-437.

18. Ishibashi H, Onozaki M, Kobayashi M, Hayashi J, Itoh H, Chiba T. Gas holdup in slurry bubble column reactors of a 150 t/d coal liquefaction pilot plant process. Fuel. 2001;80:655664.

19. Jin H, Liu D, Yang S, He G, Guo Z, Tong Z. Experimental Study of Oxygen Mass Transfer Coefficient in Bubble Column with High Temperature and High Pressure. Chemical Engineering \& Technology. 2004;27:1267-1272.

20. Lin TJ, Tsuchiya K, Fan LS. Bubble flow characteristics in bubble columns at elevated pressure and temperature. AIChE Journal. 1998;44:545-560.

21. Luo X, Lee DJ, Lau R, Yang G, Fan LS. Maximum stable bubble size and gas holdup in highpressure slurry bubble columns. AIChE Journal. 1999;45:665-680.

22. Pjontek D, Parisien V, Macchi A. Bubble characteristics measured using a monofibre optical probe in a bubble column and freeboard region under high gas holdup conditions. Chemical Engineering Science. 2014;111:153-169.

23. Schäfer R, Merten C, Eigenberger G. Bubble size distributions in a bubble column reactor under industrial conditions. Experimental Thermal and Fluid Science. 2002;26:595-604. 
24. Yang GQ, Fan LS. Axial liquid mixing in high-pressure bubble columns. AIChE Journal. 2003;49:1995-2008.

25. Yang Z, Li M, Peng B, Lin M, Dong Z, Ling, Y. Interfacial Tension of CO2 and Organic Liquid under High Pressure and Temperature. Chinese Journal of Chemical Engineering. 2014;22:1302-1306.

26. NIST, National Institute of Standards and Technology, http://webbook.nist.gov/chemistry/.

27. Cooper JR, Dooley RB. IAPWS Release on Surface Tension of Ordinary Water Substance. 1994:4.

28. Yi-Ling T, Yan-Fan X, Hong-Xu Z, Xi-Jing D, Xiao-Wen R, Feng-Cai Z. Interfacial Tensions between Water and Non-polar Fluids at High Pressures and High Temperatures. Acta. Phys. Chim. Sin. 1997;13:89-95.

29. Sugden S. The determination of surface tension from the maximum pressure in bubbles part I. J. Chem. Soc. Trans. 1922;121:858-866.

30. Blaisdell E. The physical properties of fluid interfaces of large radius of curvature. J. Math. Phys. Inst. Tech. 1940;19:186.

31. Princen HM, Zia IYZ, Mason SG. Measurement of Interfacial Tension from the Shape of a Rotating Drop. Journal of Colloid and Interface Science. 1966;23:99-107.

32. Moradi B, Awang M, Bashir A, Sabil KM. Effects of alcohols on interfacial tension between carbon dioxide and crude oil at elevated pressures and temperature. Journal of Petroleum Science and Engineering. 2014;121:103-109. 
33. Sarikhani K, Jeddi K, Thompson RB, Park CB, Chen P. Effect of pressure and temperature on interfacial tension of poly lactic acid melt in supercritical carbon dioxide. Thermochimica Acta. 2015;609:1-6.

34. Garrido JM, Cifuentes L, Cartes, M, Segura H, Mejía A. High-pressure interfacial tensions for nitrogen+ethanol, or hexane or 2-methoxy-2-methylbutane: A comparison between experimental tensiometry and Monte Carlo simulations. The Journal of Supercritical Fluids. 2014;89:78-88.

35. Badens E, Boutin O, Charbit G. Laminar jet dispersion and jet atomization in pressurized carbon dioxide. The Journal of Supercritical Fluids. 2005;36:81-90.

36. Chhetri AB, Watts KC. Surface tensions of petro-diesel, canola, jatropha and soapnut biodiesel fuels at elevated temperatures and pressures. Fuel. 2013;104:704-710.

37. Cumicheo C, Cartes M, Segura H, Müller EA, Mejía A. High-pressure densities and interfacial tensions of binary systems containing carbon dioxide $+n$-alkanes: (n-Dodecane, $n$ tridecane, n-tetradecane). Fluid Phase Equilibria. 2014;380:82-92.

38. Georgiadis A, Llovell F, Bismarck A, Blas FJ, Galindo A, Maitland GC, Trusler JPM, Jackson G. Interfacial tension measurements and modelling of (carbon dioxide + alkane) and (carbon dioxide + water) binary mixtures at elevated pressures and temperatures. The Journal of Supercritical Fluids. 2010;55:743-754.

39. Jaeger PT, Eggers R. Interfacial properties at elevated pressures in reservoir systems containing compressed or supercritical carbon dioxide. The Journal of Supercritical Fluids 2012;66:80-85. 
40. Khosharay S, Varaminian F. Experimental and modeling investigation on surface tension and surface properties of $(\mathrm{CH} 4+\mathrm{H} 2 \mathrm{O}),(\mathrm{C} 2 \mathrm{H} 6+\mathrm{H} 2 \mathrm{O}),(\mathrm{CO} 2+\mathrm{H} 2 \mathrm{O})$ and $(\mathrm{C} 3 \mathrm{H} 8+\mathrm{H} 2 \mathrm{O})$ from $284.15 \mathrm{~K}$ to $312.15 \mathrm{~K}$ and pressures up to $60 \mathrm{bar}$. International Journal of Refrigeration. 2014;47:26-35.

41. Sarmadivaleh $M, A l-Y a s e r i ~ A Z$, Iglauer S. Influence of temperature and pressure on quartz-water-CO2 contact angle and $\mathrm{CO} 2-$ water interfacial tension. Journal of Colloid and Interface Science. 2015;441:59-64.

42. Seifried B, Temelli F. Interfacial tension of marine lipids in contact with high pressure carbon dioxide. The Journal of Supercritical Fluids. 2010;52:203-214.

43. Melchior T, Bläsing M, Pütz G, Müller M. Surface tension measurements of coal ash slags under reducing conditions at elevated pressures. Fuel. 2011;90:280-287.

44. Bashforth F, Adams JC. An attempt to test the theories of capillary action: by comparing the theoretical and measured forms of drops of fluid. University Press. 1883.

45. Berry JD, Neeson MJ, Dagastine RR, Chan DYC, Tabor RF. Measurement of surface and interfacial tension using pendant drop tensiometry. Journal of Colloid and Interface Science. 2015.

46. Andreas JM, Hauser EA, Tucker WB, Boundary tension by pendant drops. J. Phy. Chem. 1938;42:1001-1019.

47. Fordham S. On the calculation of surface tension from measurements of pendant drops. Proc. R. Soc. Lond. A. 1948;194:1-16. 
48. Stauffer CE. The Measurement of Surface Tension by the Pendant Drop Technique. The Journal of Physical Chemistry. 1965;69:1933-1938.

49. Cumicheo C, Cartes M, Segura H, Müller EA, Mejía A. High-pressure densities and interfacial tensions of binary systems containing carbon dioxide+n-alkanes: (n-Dodecane, ntridecane, n-tetradecane). Fluid Phase Equilibria. 2014;380:82-92.

50. Misak MD, Equations for determining $1 / \mathrm{H}$ versus $\mathrm{S}$ values in computer calculations of interfacial tension by the pendent drop method. Journal of Colloid and Interface Science. 1968;27:141-142.

51. Saad SMI, Policova Z, Neumann AW. Design and accuracy of pendant drop methods for surface tension measurement. Colloids and Surfaces A: Physicochemical and Engineering Aspects. 2011;384:442-452.

52. Hoorfar M, Neumann A. Recent progress in Axisymmetric Drop Shape Analysis (ADSA). Advances in Colloid and Interface Science. 2006;121:25-49.

53. Padday JF, Pitt AR. The Stability of Axisymmetric Menisci. 1973;275:489-528.

54. Poling BE, Prausnitz JM, O'Connell JP. The Properties of Gases and Liquids 5th ed. 2001: McGraw-Hill. 803.

55. Hugill JA, Van Welsenes AJ. Surface Tension: A Simple Correlation for Natural Gas + Condensate Systems Fluid phase equilibria. 1986;29:383-390.

56. Sidgwick NV, Bayliss NS. The Parachor of Co-ordinated Hydrogen in the Orthosubstituted Phenols. J. Chem. Soc. 1930:2027-2034. 


\section{Figure captions}

Figure 1. Representation of an axially symmetrical hanging drop and its characteristic dimensions. $^{45}$

Figure 2. Simplified diagram of the pilot used for the measurement of surface tension.

Figure 3:. Sapphire flange allowing the visualization of the capillary and the formed drops.

Figure 4. Results of surface tension measurements in Water/Nitrogen system. Continuous feature: NIST data at saturation. ${ }^{26,27} \Delta$ : Measurements under unsaturated conditions. $\diamond$ : Measurements under saturated conditions. $\square$ : Results of Yi-Ling et al. ${ }^{28}$

Figure 5. Influence of the progressive saturation of the medium. water/nitrogen system. Operating conditions: $\mathrm{P}=30 \mathrm{MPa}-\mathrm{T}=300^{\circ} \mathrm{C}$.

Figure 6. Results of surface tension measurements on water-phenol / nitrogen system as a function of the phenol mass fraction. $\Delta$ with strokes: Measurements under unsaturated conditions. $\diamond:$ Measurements under saturated conditions.

Figure 7. Comparison between experimental values at saturation and the prediction of Eq. 12. 

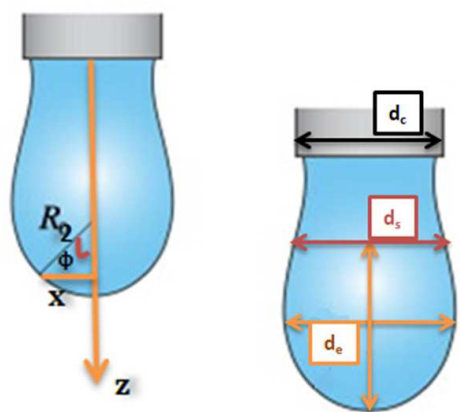

Figure 1. Representation of an axially symmetrical hanging drop and its characteristic dimensions. ${ }^{45}$ 


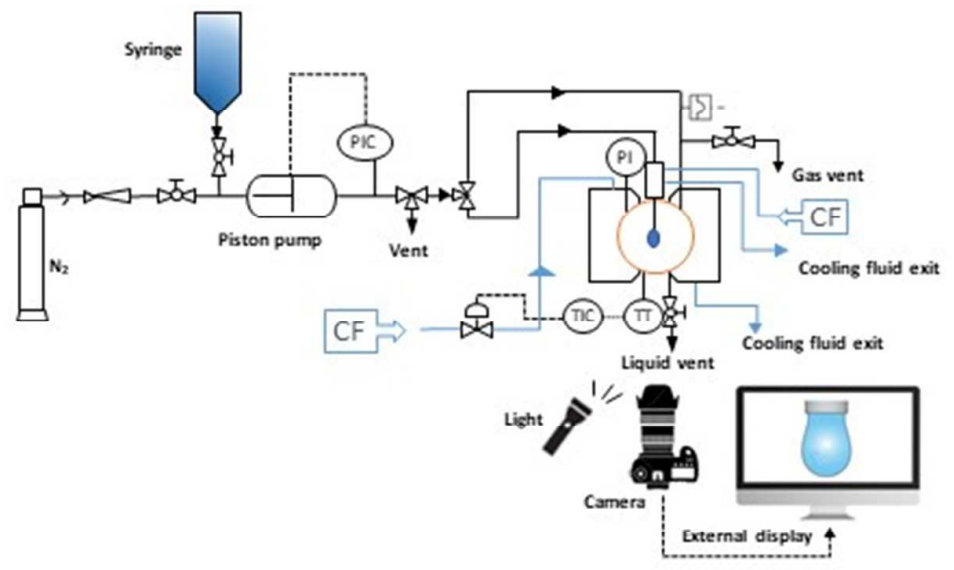

Figure 2. Simplified diagram of the pilot used for the measurement of surface tension. 


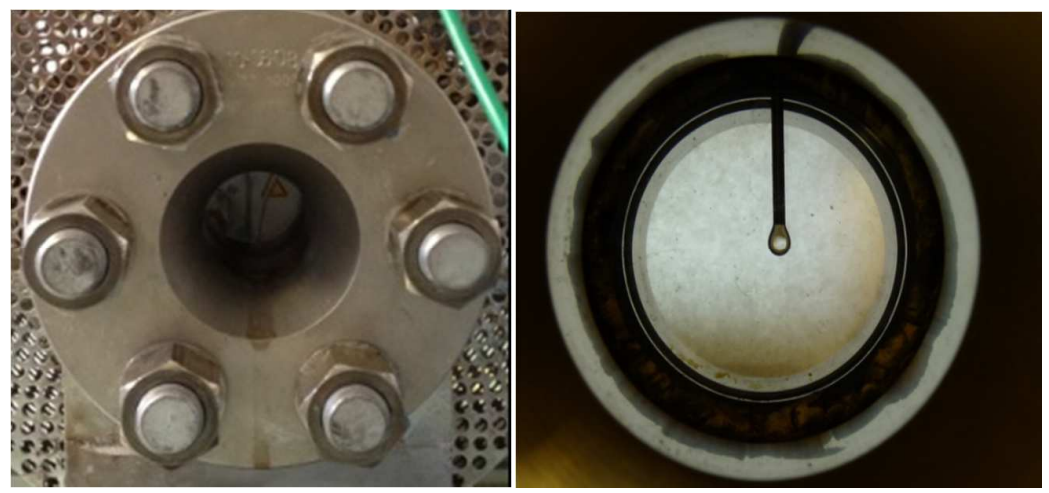

Figure 3. Sapphire flange allowing the visualization of the capillary and the formed drops. 


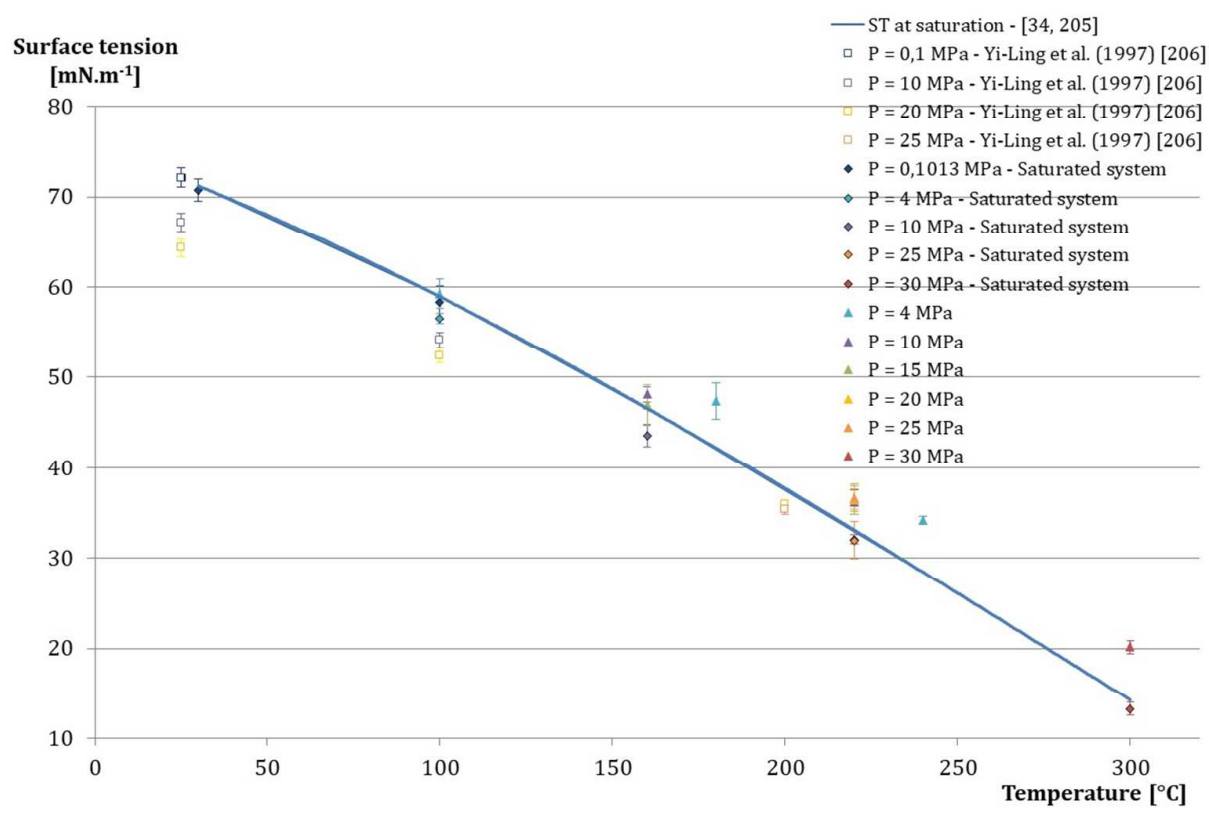

Figure 4. Results of surface tension measurements in Water/Nitrogen system. Continuous

feature: NIST data at saturation. ${ }^{26,27} \Delta$ : Measurements under unsaturated conditions. $\diamond$ :

Measurements under saturated conditions. $\square$ : Results of Yi-Ling et al. ${ }^{28}$ 


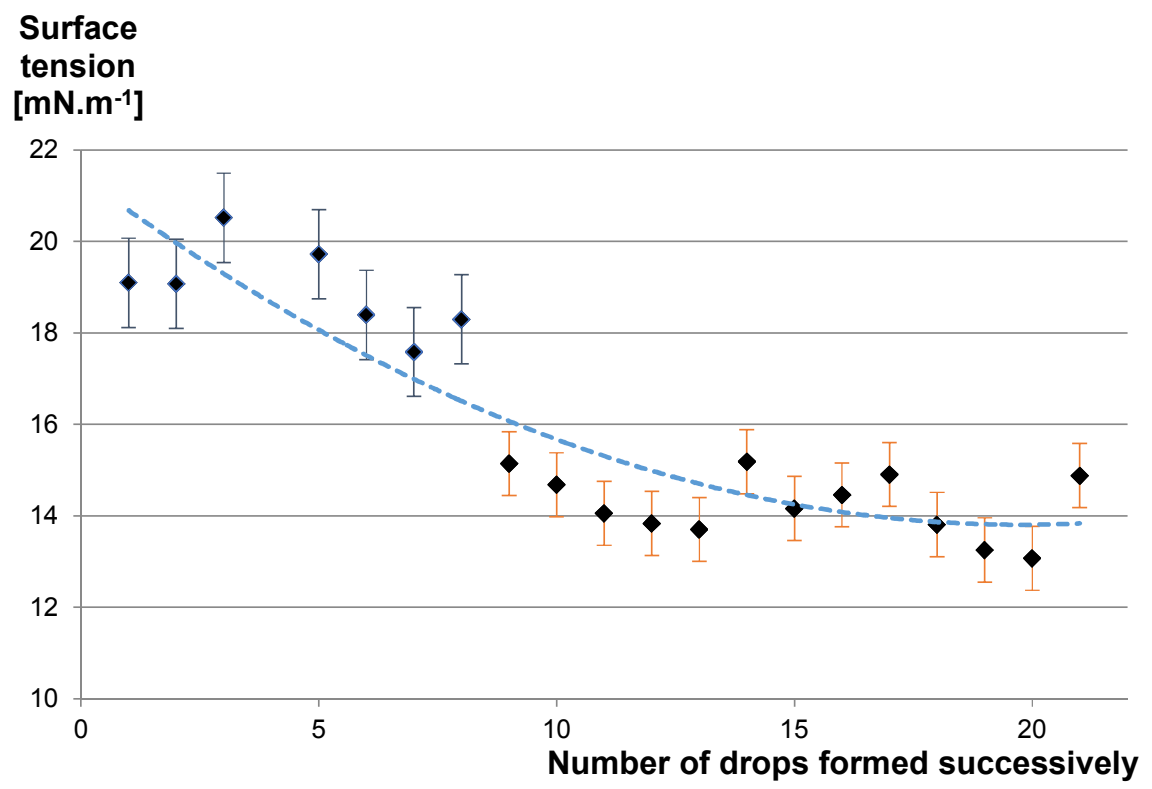

Figure 5. Influence of the progressive saturation of the medium. water/nitrogen system.

Operating conditions: $\mathrm{P}=30 \mathrm{MPa}-\mathrm{T}=300^{\circ} \mathrm{C}$. 


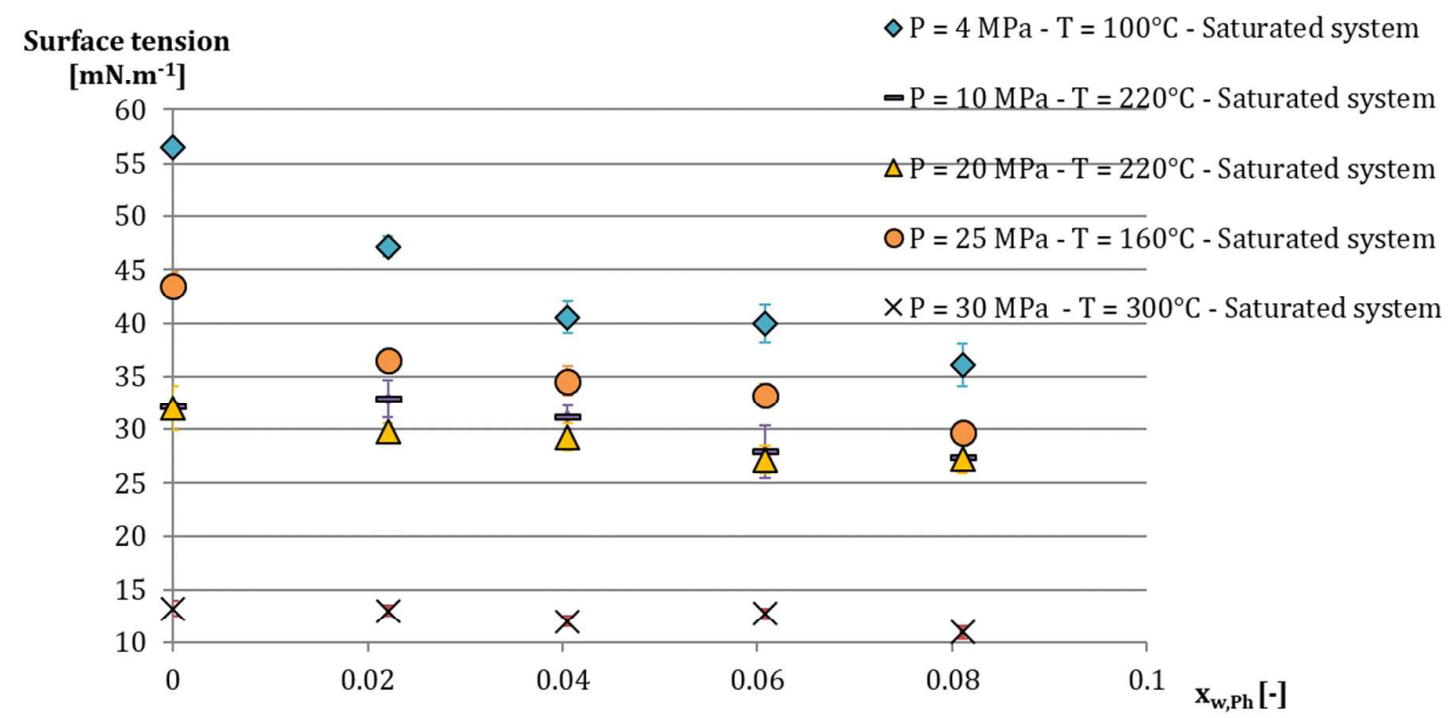

Figure 6. Results of surface tension measurements on water-phenol / nitrogen system as a function of the phenol mass fraction. $\Delta$ with strokes: Measurements under unsaturated conditions. $\diamond:$ Measurements under saturated conditions. 


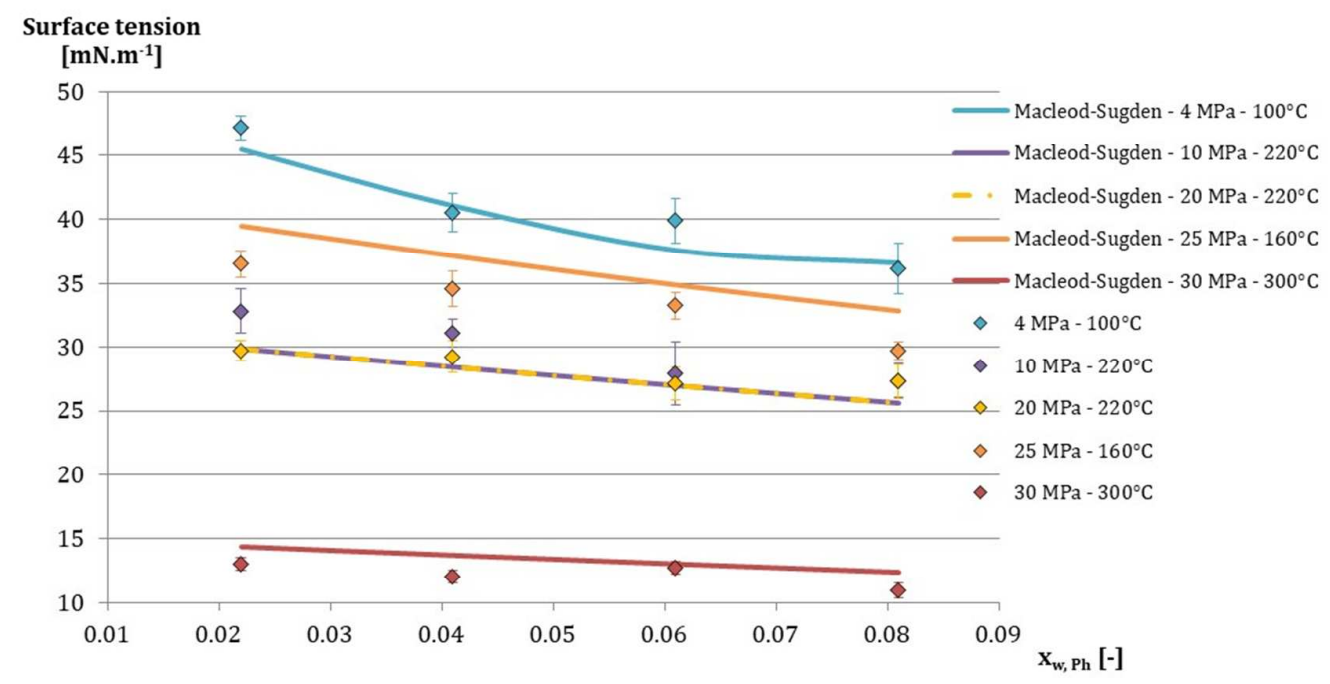

Figure 7. Comparison between experimental values at saturation and the prediction of Eq.

12. 


\begin{tabular}{ccccc}
\hline Operating conditions & $\sigma_{\mathrm{L}}$ Test I & $\boldsymbol{\sigma}_{\mathrm{L}}$ Test II & Standard deviation & Uncertainty \\
\hline $4 \mathrm{MPa}-240^{\circ} \mathrm{C}$ & $34.1 \pm 0.5$ & $35.2 \pm 0.7$ & 0.7 & 2.2 \\
\hline $15 \mathrm{MPa}-160^{\circ} \mathrm{C}$ & $46.3 \pm 1.5$ & $46.9 \pm 2.2$ & 0.4 & 0.9 \\
\hline
\end{tabular}

Table 1. Results of rReproducibility Tests (Water/Nitrogen System) 


\begin{tabular}{cc}
\hline Parameter & Value \\
\hline $\boldsymbol{\delta}_{11}$ & 1.16 \\
$\boldsymbol{\delta}_{22}$ & 80.49 \\
$\boldsymbol{\delta}_{21}$ & -1.95 \\
$\boldsymbol{\delta}_{12}$ & -1.95 \\
$\mathrm{C}$ & 3.36
\end{tabular}

Table 2. Interaction Parameters and Exponent of the Macleod-Sugden Equation. 1: Water. 2:

Phenol 

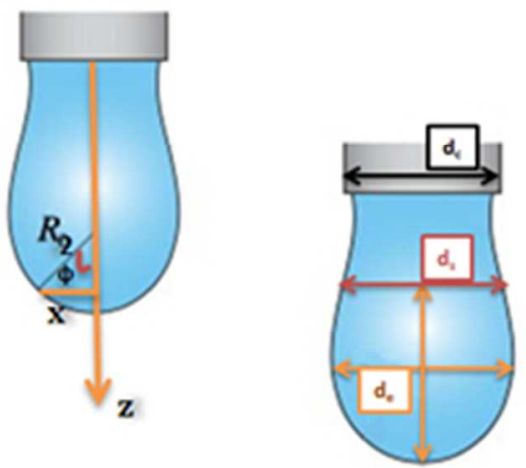

Figure 1: Representation of an axially symmetrical hanging drop and its characteristic dimensions.45 $102 \times 71 \mathrm{~mm}(76 \times 76 \mathrm{DPI})$ 


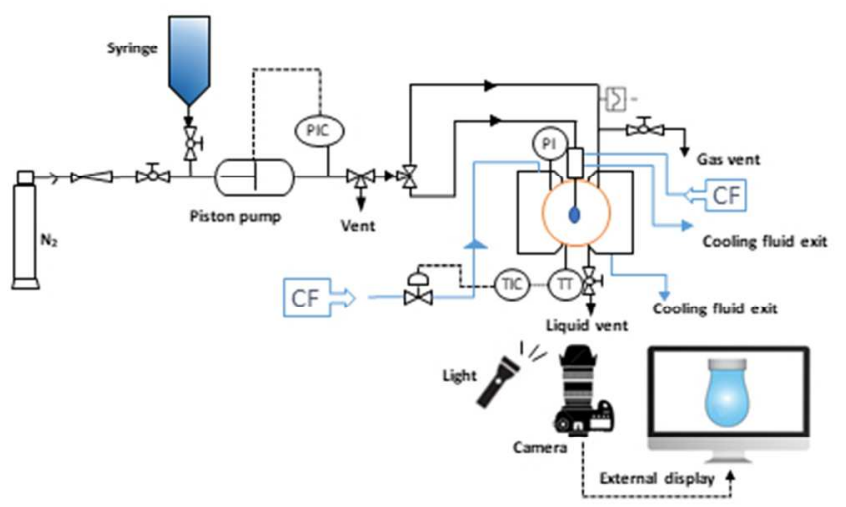

Figure 2: Simplified diagram of the pilot used for the measurement of surface tension.

$179 \times 89 \mathrm{~mm}(96 \times 96 \mathrm{DPI})$ 


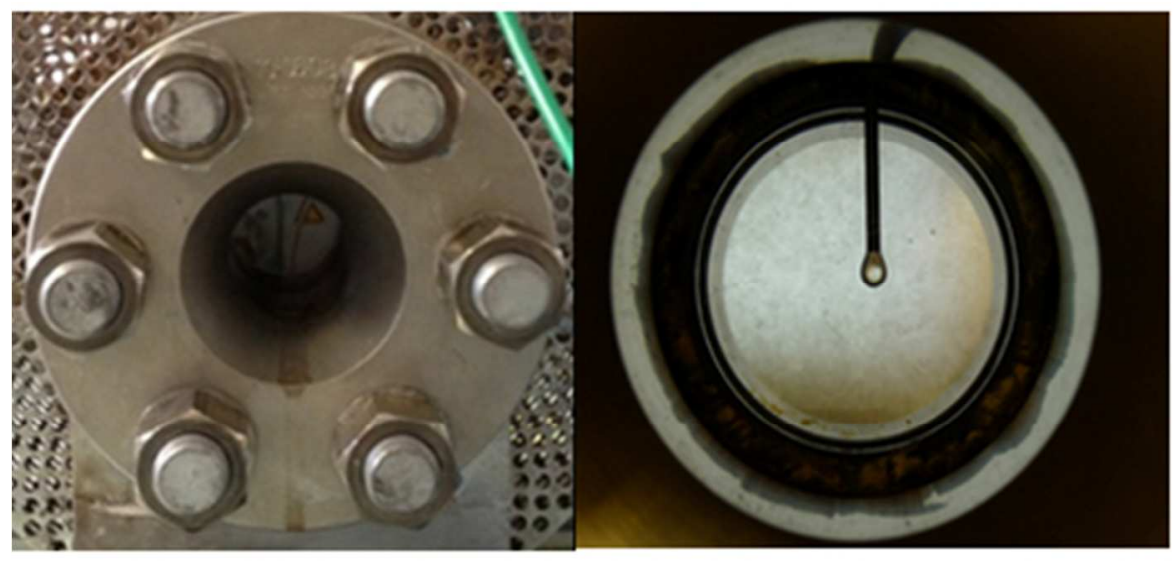

Figure 3: Sapphire flange allowing the visualization of the capillary and the formed drops. $149 \times 72 \mathrm{~mm}(76 \times 76 \mathrm{DPI})$ 


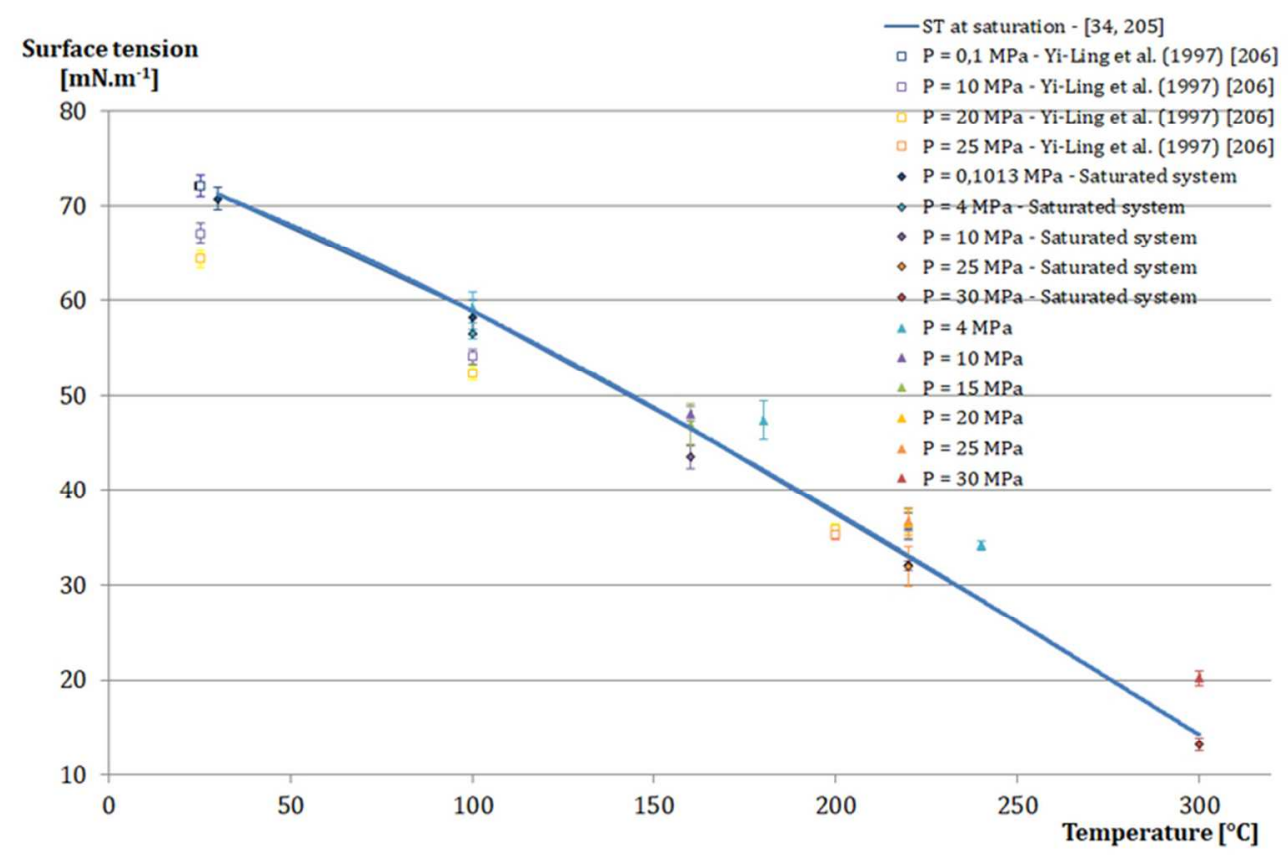

Figure 4: Results of surface tension measurements in Water/Nitrogen system. Continuous feature: NIST data at saturation.26,27 $\Delta$ : Measurements under unsaturated conditions. $\diamond:$ Measurements under saturated conditions. $\square$ : Results of Yi-Ling et al.28

$324 \times 215 \mathrm{~mm}(64 \times 64$ DPI $)$ 


\section{Surface tension $\left[\mathrm{mN} \cdot \mathrm{m}^{-1}\right]$}

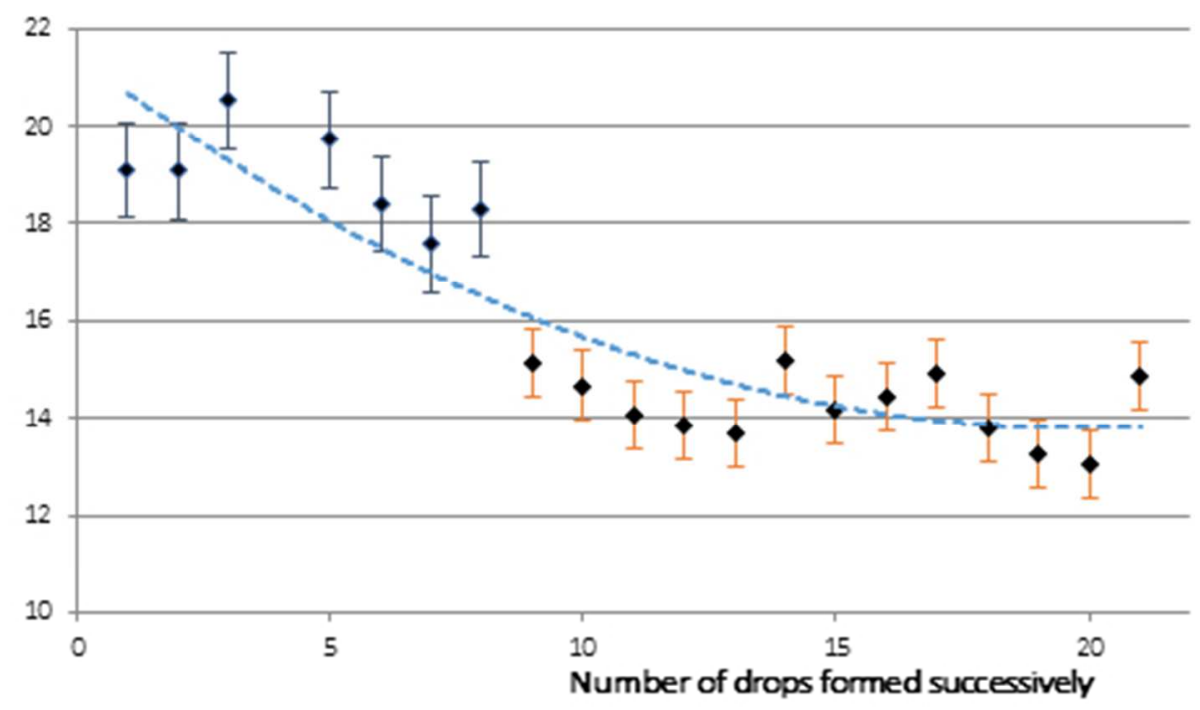

Figure 5: Influence of the progressive saturation of the medium. water/nitrogen system. Operating conditions: $\mathrm{P}=30 \mathrm{MPa}-\mathrm{T}=300^{\circ} \mathrm{C}$.

$160 \times 110 \mathrm{~mm}(76 \times 76 \mathrm{DPI})$ 


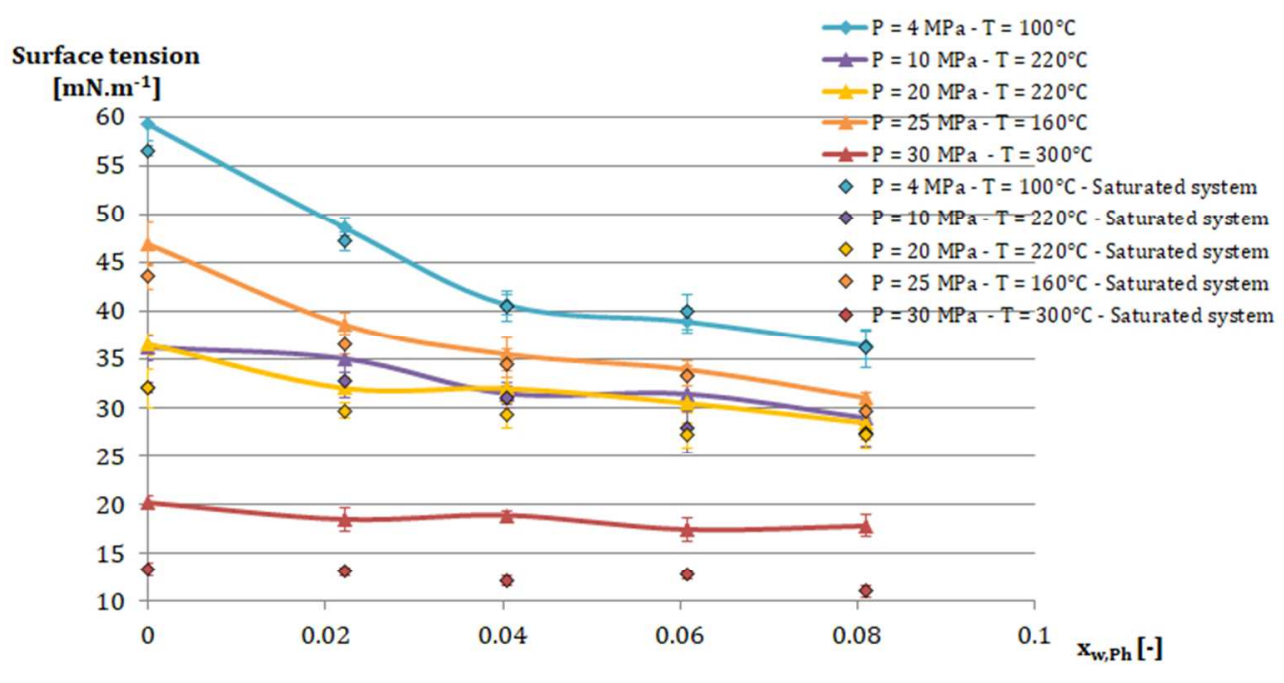

Figure 6: Results of surface tension measurements on water-phenol / nitrogen system as a function of the phenol mass fraction. $\Delta$ with strokes: Measurements under unsaturated conditions. $\diamond:$ Measurements under saturated conditions.

$260 \times 140 \mathrm{~mm}(76 \times 76 \mathrm{DPI})$ 


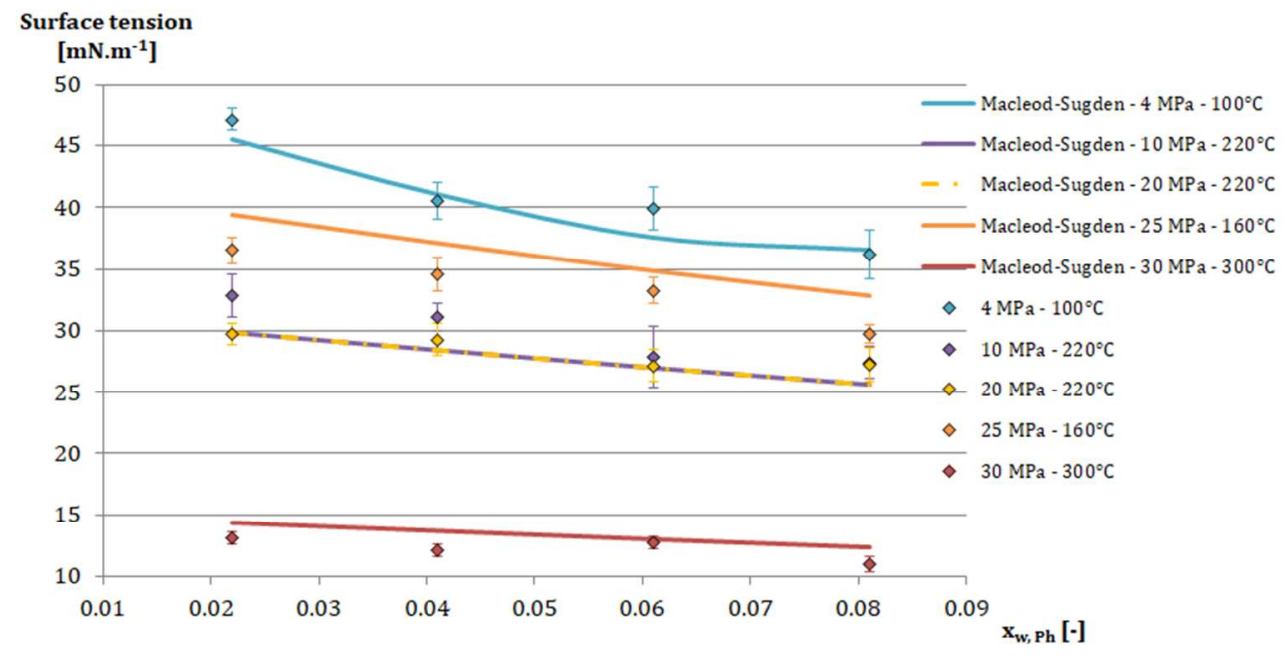

Figure 7: Comparison between experimental values at saturation and the prediction of equation 12 $284 \times 143 \mathrm{~mm}(76 \times 76 \mathrm{DPI})$ 